\title{
UNIVERSIDAD PARA PERSONAS MAYORES, UNA EXPERIENCIA DE GERONTOLOGÍA EDUCATIVA
}

\section{UNIVERSITY FOR THE ELDERLY, AN EDUCATIONAL GERONTOLOGY EXPERIENCE}

Fecha recepción: 15 de marzo de 2019 / fecha aceptación: 2 de julio de 2019

\author{
Sandra Sandoval ${ }^{1}$ y Karina Tejada ${ }^{2}$
}

Cómo citar este artículo:

Sandoval, S. y Tejada, K. (2019).Universidad para personas mayores, una experiencia de gerontología educativa. Revista Pensamiento y Acción Interdisciplinaria, 5(1), 95-107. DOI: http://doi.org/10.29035/pai.5.1.95

\section{Resumen}

El proyecto "Universidad para Personas Mayores", tiene el objetivo de promover la Calidad de Vida de las Personas Mayores, favoreciendo el envejecimiento activo a través de una oferta académica centrada en las necesidades de esta etapa evolutiva, pretendiendo además el desarrollo de habilidades teórico-prácticas ${ }^{3}$ (Escuela de Psicología, 2017), para la elaboración de un proyecto de innovación y/o emprendimiento. Etapas del proyecto: Diagnóstico para el levantamiento de las necesidades y preferencias de formación en las personas mayores; Elaboración de propuesta curricular; Apertura de matrícula, inicio de clases; Monitoreo; Evaluación y seguimiento del Proyecto. En la actualidad este proyecto está en la etapa de ejecución.

Palabras claves: Envejecimiento activo, calidad de vida y educación gerontológica.

\section{Abstract}

The project "University for the Elderly" has the objective of promoting the Quality of Life, favoring active aging through an academic offer focused on the needs of this evolutionary stage, also aiming at the development of theoretical skills- practices (School of Psychology, 2017), for the elaboration of an innovation and / or entrepreneurship project. Stages: Diagnosis for the lifting of training needs and preferences in the elderly; Preparation of curricular proposal; Registration opening, start of classes; Monitoring; Evaluation and monitoring of the Project. Currently this project is in the execution stage.

Keywords: Active aging, quality of life, educational gerontology

\footnotetext{
1 Chilena. Directora de Proyecto Universidad para Personas Mayores. Psicóloga, Terapeuta de Familia y Parejas, Doctoranda en Psicología Universidad de Buenos Aires. Académica de la Escuela de Psicología de la Universidad Santo Tomas, Antofagasta, Chile. E-mail: electrónico ssandovalpasten@gmail.com.

2 Chilena. Asesora Pedagógica Universidad para Personas Mayores. Profesora de Filosofía, Psicóloga Educacional, Magister en Educación. Académica de la Escuela de Psicología de la Universidad Santo Tomas, Antofagasta, Chile. E-mail: karinatejadac@gmail.com.

3 Este artículo da cuenta del proceso de ejecución, en su fase intermedia, del Proyecto de Vinculación con el Medio, "Universidad para Personas Mayores", de la Escuela de Psicología, sede Antofagasta, aprobado por Universidad Santo Tomás, en el 2017. Código de proyecto nro. 0000029416.
} 


\section{Antecedentes Generales}

El envejecimiento demográfico es un fenómeno en la población que se está produciendo a pasos agigantados. En Chile, en el año 2017 1,1 de cada 10 personas de la población eran personas mayores de 64 años, para el año 2050 se espera que se incremente este número a un 2,2 de cada 10 personas de la población (INE, 2018). Un efecto importante de esta transformación demográfica, será el incremento de las demandas sociales y económicas de las PM, lo que probablemente provocará un cambio en las políticas públicas con la finalidad de redistribuir los recursos económicos para atender las demandas de esta población (CEPAL, 2017).

En estudio realizado el 2011, se establece la importancia para las personas mayores (PM) de sentirse satisfecho con el modo de usar su tiempo, su nivel de actividad y con su oportunidad de participar en la comunidad (Urzúa, Bravo, Ogalde \& Vargas, 2011). Se establece también que la Autoaceptación se favorece, en la medida que las PM valoran sus capacidades, habilidades y aceptan sus limitaciones, esto mejora su capacidad para solucionar los problemas de la vida diaria, así como el sentirse satisfecho con las actividades que realiza (Urzúa, Bravo, Ogalde \& Vargas, 2011). Esto permitiría que las PM alcancen sus metas u objetivos, permitiendo su adaptación a la etapa del ciclo vital (Sanjuán, Pérez \& Bermúdez, 2000). Considerando además, que a partir de la jubilación, las PM, sufren la pérdida del poder, autonomía y del rol que ocupaba en la sociedad, surge la necesidad de elaborar un nuevo proyecto de vida (Gómez, Villegas De Posada, Barrera \& Cruz, 2007).

En este contexto la Escuela de Psicología, sede Antofagasta, junto al Centro de Investigación en Gerontología Aplicada (CIGAP) y las Escuelas de Ingeniería Comercial, Enfermería, Nutrición y Dietética, Derecho, Kinesiología, área de Lenguaje de Formación General y Formación de Identidad de la UST sede Antofagasta, desarrollan el proyecto de 'Universidad para Personas Mayores' (UPM), con enfoque de derechos y cuyo objetivo es promover Calidad de Vida, favoreciendo el envejecimiento activo a través de una oferta académica centrada en las necesidades de esta etapa evolutiva, que está en coherencia con los ejes estratégicos del SENAMA, pretendiendo el desarrollo de habilidades teórico-prácticas (Escuela de Psicología, 2017), el que se concretará con un trabajo final en el que los participantes presentarán un proyecto de innovación y emprendimiento a la comunidad. La cobertura es de 60 PM y es financiado con fondos de la subdirección de Vinculación con el Medio de la UST.

Un sello distintivo del proyecto UPM, es el favorecer el desarrollo de productos por parte de las PM, a través del aprendizaje de metodologías que permitan elaborar proyectos innovadores. 


\section{Etapas del Proyecto}

\section{Etapa 1. Diagnóstico 4}

Para el Diagnóstico se realizaron 4 grupos focales a PM de agrupaciones de juntas de vecinos y del Programa Más Adultos Mayores Autovalentes. Algunos de los resultados se pueden apreciar en la Tabla 1.

Tabla 1. Resultados Grupos Focales

\begin{tabular}{|l|l|}
\hline Motivación para participar en la UPM & $\begin{array}{l}\text { Cursos o asignaturas distintas a las } \\
\text { propuestas }\end{array}$ \\
\hline $\begin{array}{l}\text { Recreación y Aprendizaje } \\
\text { Emprendimiento y ayuda social para otros. } \\
\text { Participación en conjunto con estudiantes de } \\
\text { pregrado }\end{array}$ & $\begin{array}{l}\text { Derechos de las Personas Mayores } \\
\text { Turismo (capacitarse y/o viajar) }\end{array}$ \\
Interés por trabajar en equipos de proyecto. & $\begin{array}{l}\text { Manualidades } \\
\text { Computación básica y avanzada } \\
\end{array}$ \\
& $\begin{array}{l}\text { Actividad física } \\
\text { Hidroponía y cultivo }\end{array}$ \\
\hline
\end{tabular}

Una vez realizados los grupos focales se elaboró una encuesta, a partir de las categorías levantadas. La encuesta fue aplicada a 189 PM. Los principales resultados de esta encuesta, indican que el tiempo de duración de las clases, debería ser de dos a tres horas con un $82 \%$ de las preferencias, un 49\% prefiere asistir 2 días a la semana y un $32 \%$ le gustaría asistir 3 días a la semana a clases. El $86 \%$ de las PM que contestaron la encuesta, les gustaría participar en actividades que incluyan el intercambio con estudiantes de pregrado. Por otro lado, el 94\% de las PM les gustaría trabajar con otras PM en equipos de trabajo. En las figuras 1 y 2 que se presentan a continuación, se observan los temas de preferencia de las PM.

Entre los temas básicos del curso, ¿Cúales de los siguientes incorporaría?

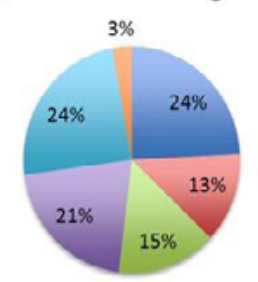

Figura 1

\section{¿Que temas considera que debiesen incluirse en los electivos?}
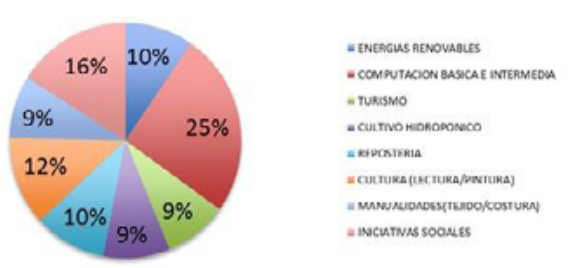

Figura 2

4 Diagnóstico Diseñado y Aplicado por el Doctor Jerome Flores Jara, en colaboración de los Estudiantes de la Escuela de Psicología y la Asistente académica de UPM Patricia Navarrete Urra. 


\section{Etapa 2.}

\section{Propuesta curricular Universidad para Personas Mayores ${ }^{5}$}

\section{Enfoque pedagógico}

Esta propuesta se pensó desde la experiencia de enfoques educativos y socioeducativos, que indican y refuerzan principios de aprendizajes que convierten la experiencia educativa en algo significativo, donde, como señala Gómez, C y Coll, C., (1994). "Todo conocimiento se construye en estrecha interrelación con los contextos en los que se usa, y que, por lo tanto, no es posible separar los aspectos cognitivos, emocionales y sociales presentes en el contexto en el que se actúa" (p.5). Se consideró un proceso formativo y de estudio, centrado en los fundamentos del modelo de aprendizaje, declarado en la Pedagogía Gerontológica, como disciplina que promueve el desarrollo personal y social de la PM, capacitándola para ser miembro contributivo y receptivo del entramado social en el que vive y se desarrolla (García, N. 2007, p.8).

Desde esta mirada es que se establecieron cuatro aspectos orientadores, Según Bermejo, L (2012), en primer lugar, a Enfoque enseñanza-aprendizaje (E-A) constructivista. En segundo lugar, el espacio pedagógico, que favorezca el fortalecimiento de competencias: conceptual (saber), procedimental (hacer), actitudinal (querer). En tercer lugar, al rol del educador; como 'mediador' entre aspectos objetivos (contenidos) y subjetivos (interpretación y construcción del conocimiento). Bajo esta premisa, es que se planteó como número ideal de estudiantes de 15, con un máximo de 20, por grupo.

\section{Orientaciones en el diseño de cursos}

Para la fase de diseño, se les propuso a los docentes, contemplar algunas directrices asociadas a la formulación de objetivos y contenidos, metodología y evaluación en un formato estándar. Se sugirió que la metodología promoviera un aprendizaje activo del estudiante, el cual necesariamente vivencie el trabajo en grupo desde el enfoque de aprendizaje cooperativo (García, A. J., \& Troyano Rodríguez, Y. 2010).

La selección y activación de conocimientos declarativos (saber), procedimentales (saber hacer) y actitudinales (saber ser) se debía dar en función de un conocimiento sobre el contexto: a) condiciones y características de los adultos, b) condiciones del área y/o actividad requerida, c) condiciones de la didáctica a utilizar.

5 Propuesta elaborada por Karina Tejada Campos, Profesora de Filosofía, Psicóloga Educacional, Magister en Educación, en su rol de Asesora Pedagógica.

6 Propuesta elaborada por Karina Tejada Campos, Profesora de Filosofía, Psicóloga Educacional, Magister en Educación, en su rol de Asesora Pedagógica. 
La evaluación, considerada como consustancial al proceso de enseñanza-aprendizaje (E-A), se propuso que fuera fundamentalmente formativa, esto implicó que fuera considerada como un proceso unido a las actividades diarias de enseñanza, como una parte natural de la E-A. Desde la concepción de la evaluación auténtica, entendiendo que la evaluación se planifica y se desarrolla al mismo tiempo que se enseña (Black \& William, 1998). Según esto se propusieron algunas consideraciones:

Tabla 2. Consideraciones evaluativas

1. Evaluar las reales competencias de las PM a partir de la información que aportan sus desempeños durante el proceso.

2. Constituirse como un proceso cooperativo, por tanto, puede considerar modalidades dependiendo del agente evaluador, pudiendo ser las PM quienes se autoevalúan, y/o sean evaluados por sus pares (coevaluación) y por el profesor (heteroevaluación) y este, a la vez, aprende de y con las PM.

3. Se debe privilegiar la actividad de las PM, sus características y conocimientos previos y los contextos donde ocurre el proceso aprendizaje.

4. Se debe centrar en las fortalezas y logros de las PM, pues los ayuda a identificar lo que saben o dominan.

Propuesta elaborada por asesora pedagógica Tejada, K.

\section{Plan de estudio y programación}

Este plan estableció cursos troncales (obligatorios) y una oferta de cursos electivos, que permitieran direccionar y nutrir el eje articulador de esta propuesta que es la elaboración, por parte de los estudiantes, de proyectos de emprendimiento y/o innovación. De esta manera el curso troncal, transversal y de más larga duración es el de "Diseño de proyectos", más otros dos troncales, el de "Envejecimiento exitoso y calidad de vida" y "Formación e Identidad" (sello de nuestra institución). Los períodos considerados para su implementación comprendieron el segundo semestre del año 2017. 
Tabla 3. Distribución periodos carga académica

\begin{tabular}{|c|c|}
\hline $\begin{array}{l}\text { PERIODO 1: } 38 \text { horas pedagógicas } \\
\text { programadas }\end{array}$ & $\begin{array}{l}\text { PERIODO 2: } 38 \text { horas pedagógicas } \\
\text { programadas }\end{array}$ \\
\hline $\begin{array}{l}\text { Primeras } 7 \text { semanas: } \\
\text { del } 14 \text { de agosto al } 6 \text { de octubre }\end{array}$ & $\begin{array}{l}\text { Segundas } 7 \text { semanas: } \\
\text { del } 9 \text { de octubre al } 24 \text { de noviembre }\end{array}$ \\
\hline 2 cursos troncales: & 2 cursos troncales: \\
\hline $\begin{array}{l}\text { Diseño de proyecto I: de } 14 \text { horas } \\
\text { pedagógicas, distribuidas en } 7 \text { semanas. }\end{array}$ & $\begin{array}{l}\text { Diseño de proyecto II: de } 14 \text { horas } \\
\text { pedagógicas, distribuidas en } 7 \text { semanas. }\end{array}$ \\
\hline $\begin{array}{l}\text { Envejecimiento exitoso y calidad de vida: } \\
\text { de } 14 \text { horas pedagógicas, distribuidas en } 7 \\
\text { semanas. }\end{array}$ & $\begin{array}{l}\text { Formación e identidad: de } 14 \text { horas } \\
\text { pedagógicas, distribuidas en } 7 \text { semanas. }\end{array}$ \\
\hline & 1 curso electivo: \\
\hline 1 curso electivo: & De 10 pedagógicas, distribuido en 5 \\
\hline $\begin{array}{l}\text { De } 10 \text { horas pedagógicas, distribuido en } 5 \\
\text { semanas, iniciándose la semana tres } \\
\text { ( } 28 \text { de Agosto). }\end{array}$ & $\begin{array}{l}\text { semanas, iniciándose la semana tres } \\
\text { ( } 23 \text { de Octubre). }\end{array}$ \\
\hline
\end{tabular}

Elaborado por asesora pedagógica Tejada, K. en consideración a las propuestas de las unidades académicas colaboradoras y calendarización de actividades académicas de la institución.

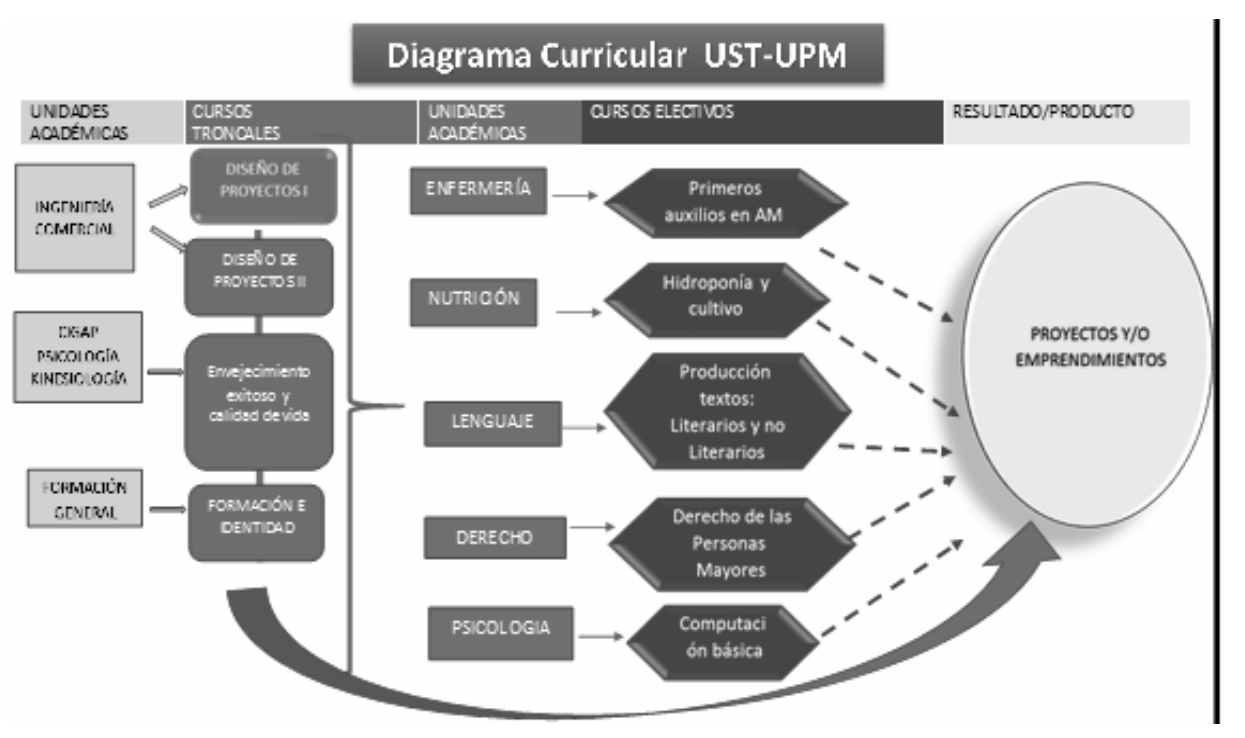

Figura 3. Articulación y participación de las distintas Unidades Académicas de Universidad Santo Tomás, sede Antofagasta en Proyecto Universidad para Personas Mayores. Este diagrama fue diseñado por la asesora pedagógica Tejada, K., en consideración a lo planteado por la Coordinación del Proyecto. 


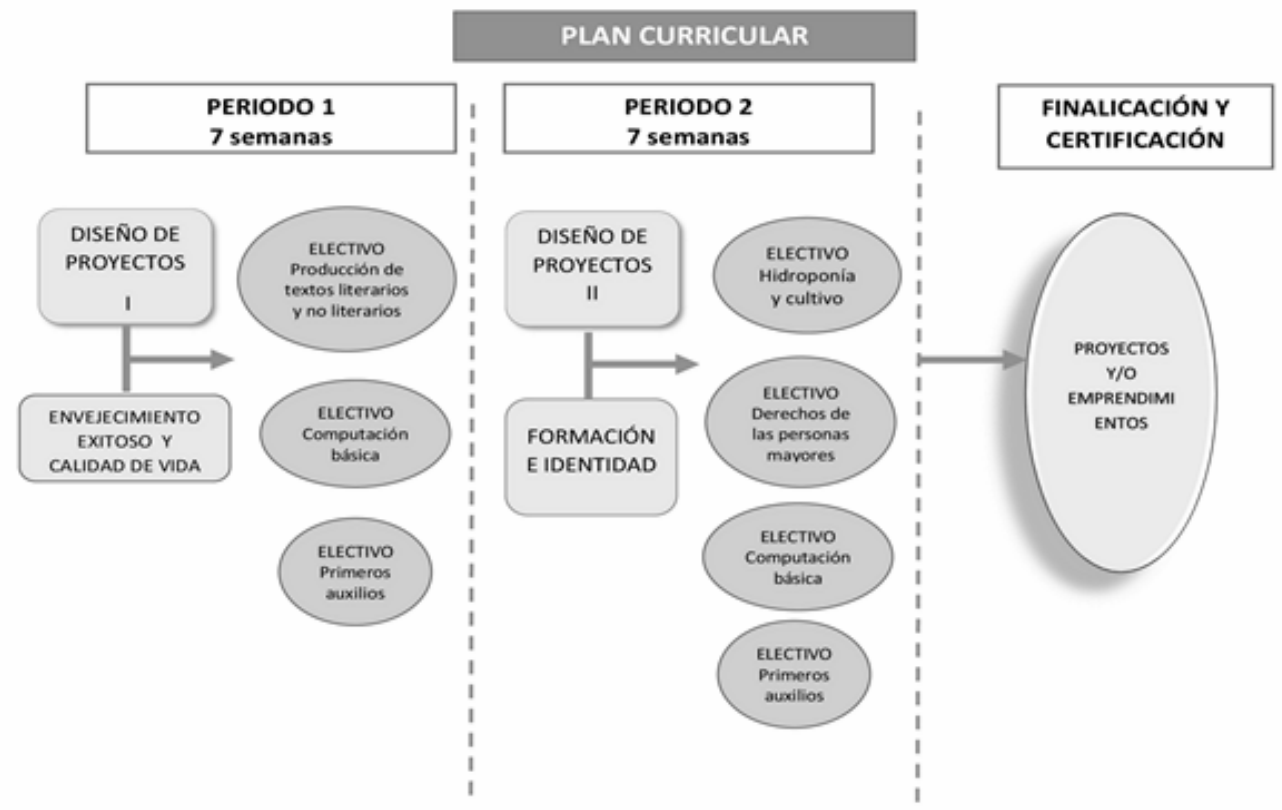

Figura 4. Elaborada por asesora pedagógica Tejada, K.

\section{Etapa 3: Apertura de Clases e Inicio de Clases}

La matrícula quedó en 60 PM, distribuidos en tres secciones de 20 Personas cada una. Del total de PM matriculados/as, asisten regularmente 52. En cuanto a la distribución por sexo se puede señalar que el 66\% de las PM que asisten a la Universidad son mujeres.

Etapa 4: Monitoreo-Análisis de Primer Período de clases. Resultados preliminares.

Con el objeto de monitorear el proceso y efectuar las mejoras pertinentes, es que se aplicaron cuatro instrumentos. Dos para evaluar aspectos del programa y dos al proceso de E-A. 


\section{Evaluación de monitoreo del Programa}

Encuesta Satisfacción de Vinculación con el Medio. Instrumento desarrollado para actividades de VM en la UST. Los principales resultados obtenidos dicen relación con que el $87 \%$ de los estudiantes de la UPM encuentra muy buena la organización de las actividades y el $94 \%$ de los estudiantes evalúan como muy buena la UPM.

SERVPERF8. Instrumento para evaluar calidad del servicio, el que presentó resultados positivos respecto a las categorías de desempeño Docente, Personal administrativo, Instalaciones y Programa en general.

\section{Evaluación de monitoreo de proceso E-A. ${ }^{9}$}

Cuestionario aplicado a Docente: Resultados de la evaluación de seguimiento al proceso de enseñanza-aprendizaje generado.

7 Proceso dirigido por el Doctor Jerome Flores Jara, en colaboración de los Estudiantes de la Escuela de Psicología y la Asistente académica de UPM Patricia Navarrete.

8 Instrumento ajustado y aplicado por el Doctor Jerome Flores Jara en colaboración de los Estudiantes de la Escuela de Psicología y la Asistente académica de UPM Patricia Navarrete.

9 Realizado por asesora pedagógica, Karina Tejada Campos, con colaboración de estudiantes de Escuela de Psicología. 
Tabla 4 Resultados cualitativos-Docentes

\section{Categoría 1. Enseñanza-Aprendizaje}

Subcategoría

Estrategias

pedagógicas

utilizadas.
Ejemplificaciones en el abordaje de contenidos. Discusiones y reflexiones grupales dirigidas. Dinámicas lúdicas, de juegos prácticos. Ejercicios de simulación. Representaciones grupales: Teatral-Audiovisual-Exponencial.
Subcategoría Característica del estudiante.
Buena actitud frente a la crítica. Comprometidos/Participativos / Reflexivos. Lograban integrar los contenidos y relacionarlos con sus experiencias individuales y subjetivas. Buena disposición. Respetuosos de las opiniones de los demás y de facilitadores.

\section{Categoría 2. Evaluación de Logros de los Estudiantes}

Participación en discusiones. Ejercicios prácticos. Retroalimentación según observaciones. Trabajo grupal de aplicación de conocimientos.

\section{Categoría 3. Tareas del mediador y/o Facilitador}

Asegura climas agradables: Desafío en el manejo eficaz de las interacciones. Ser flexibles frente a la planificación de actividades. Asegurar comprensión de los contenidos expuestos (resumir, reforzar, repetir).

\section{Categoría 4. Otras observaciones sobre proceso de aprendizaje en el aula, en el marco de este proyecto educativo}

Hacer uso adecuado de la voz (alta y clara). Resguardar recurso auditivo. Ajustar a recursos visuales (power point). Distribuir de manera equivalente en las secciones, en el mejor uso de los tiempos para la participación y la configuración de la idea de "curso". Capacitar a futuros facilitadores de aprendizaje en primeros auxilios. Mejorar disponibilidad de fuentes teóricas. 
Grupos Focales aplicados a Estudiantes UPM.

Tabla 5 Resultados cualitativos-estudiantes

\begin{tabular}{|c|c|}
\hline $\begin{array}{l}\text { Subcategoría } \\
\text { Recursos Personales. }\end{array}$ & $\begin{array}{l}\text { Ejemplificaciones en el abordaje de contenidos. Discusiones } \\
\text { y reflexiones grupales dirigidas. Dinámicas lúdicas, de juegos } \\
\text { prácticos. Ejercicios de simulación. Representaciones grupales: } \\
\text { Teatral-Audiovisual-Exponencial. }\end{array}$ \\
\hline $\begin{array}{l}\text { Subcategoría } \\
\text { dificultades frente } \\
\text { al proceso de } \\
\text { aprendizaje. }\end{array}$ & $\begin{array}{l}\text { Buena actitud frente a la crítica. Comprometidos/Participativos / } \\
\text { Reflexivos. Lograban integrar los contenidos y relacionarlos con } \\
\text { sus experiencias individuales y subjetivas. Buena disposición. } \\
\text { Respetuosos de las opiniones de los demás y de facilitadores. }\end{array}$ \\
\hline $\begin{array}{l}\text { Subcategoría } \\
\text { estrategias de } \\
\text { estudio utilizadas. }\end{array}$ & $\begin{array}{l}\text { Hacer las "tareas", leer, sacar ideas (o marcarlas) y escribirlas, leer } \\
\text { en voz alta. Juntarse en grupos a revisar la materia, a leer juntos y } \\
\text { comentar. Buscar libros complementarios en la biblioteca. Buscar en } \\
\text { diccionario palabras. }\end{array}$ \\
\hline \multicolumn{2}{|c|}{ Categoría 2. Respecto al facilitador de aprendizaje } \\
\hline $\begin{array}{l}\text { Subcategoría } \\
\text { características } \\
\text { del facilitador y } \\
\text { estrategias de } \\
\text { aprendizaje. }\end{array}$ & $\begin{array}{l}\text { Respetuosos / Cordiales/ Preocupados / Atentos. Expertos en } \\
\text { sus temas /Claros en las explicaciones. Actividades entretenidas, } \\
\text { actuar, jugar, competir en desafíos (montaña de fideos). Favorecer } \\
\text { la interacción. Se hace especial referencias a los estudiantes de } \\
\text { Psicología que cumplieron labores docentes, destacando cualidades } \\
\text { positivas. }\end{array}$ \\
\hline $\begin{array}{l}\text { Subcategoría lo que } \\
\text { se debe mejorar. }\end{array}$ & $\begin{array}{l}\text { Uso del lenguaje técnico siempre asociado a un lenguaje ordinario. } \\
\text { Operatividad de los computadores. Ofrecer (enseñar) estrategias de } \\
\text { estudio. Uso adecuado del tiempo de la clase, para la participación } \\
\text { de todos. No usar textos muy largos y con letra grande. }\end{array}$ \\
\hline
\end{tabular}

\section{Etapa 5. Evaluación y seguimiento de Proyecto ${ }^{10}$}

Esta etapa está pendiente. Se tiene planificado realizar evaluación de variables de Calidad de Vida, Deterioro Cognitivo y Depresión en PM participantes del programa; Evaluación de la Calidad del Servicio y evaluación del proceso de E-A. Respecto al seguimiento, se aplicará un plan de acompañamiento durante el 2018 para que nuestros ex-alumnos de la UPM, hagan efectiva la postulación a fondos y/o el desarrollo de sus proyectos.

10 Esta etapa está a cargo de la Directora del Proyecto y de Doctor en Psicología Social Jerome Flores Jara y el Magíster en Neuropsicología Clínica, Magíster Gerontología Clínica Miguel Ramos Henderson. 


\section{Conclusiones}

El desafío de este Proyecto es ofrecer a la población de las PM de la comuna de Antofagasta, un espacio de aprendizaje que permita un efectivo reconocimiento de las habilidades y potencialidades de crear, socializar y realizar proyectos vitales en un marco formal, con un acompañamiento real y profesional, que permita a su vez favorecer la calidad de vida y el envejecimiento activo.

En consideración a los resultados preliminares que surgen de la etapa 4 Monitoreo, nos encontramos con indicadores positivos, en cuanto a la calidad del Servicio, lo que implica para el equipo coordinador del proyecto mantener y mejorar el nivel de ejecución. Los aspectos por mejorar, dicen relación con involucrar de forma activa al resto del personal administrativo y académico de la sede de la UST, que no tienen directa relación con nuestro proyecto, pero que igualmente interactúan con los estudiantes de la UPM. Además de procurar el aseguramiento de espacios adecuados para la realización de las clases, en consideración de las características de las PM.

El foco del proceso E-A en este proyecto, como lo indican los resultados preliminares, es dar cabida a la gran motivación y actitud positiva que demuestran un gran grupo de PM, con capacidad de construir y re-construir aprendizajes, donde los aspectos más subjetivos de las experiencias memorables de ellos (procesos de intersubjetividad y contextos socio-históricos), se vuelve elementos objetivossignificativos de aprendizajes cognitivos, emocionales y sociales. Este proyecto, además, refuerza la autonomía de la PM, con capacidad de decisión, de organización, de pro-actividad, pero también de establecer sus demandas, opiniones y juicios respecto a sus requerimientos y expectativas. Nuestro desafío en cuanto a proceso de E-A, es contar con instrumentos de evaluación de aprendizajes, que den cuenta de manera objetiva de los logros alcanzados.

Finalmente, la evaluación de este programa y su impacto, será realizado y socializado una vez terminado el proyecto, permitiendo con ello, tomar decisiones en torno a la proyección de acciones de Vinculación con el Medio y convertirnos en una red de apoyo y promoción de la Calidad de Vida de las PM de la comuna y tal vez un modelo replicable para el resto del país. 


\section{Referencias}

Álvarez, I. (2005). Evaluación como situación de aprendizaje o evaluación auténtica. Perspectiva Educacional, Formación de Profesores, 45, 45-68

Bermejo, L. (2012). Envejecimiento activo, pedagogía gerontológica y buenas prácticas socioeducativas con personas adultas mayores. Educación social. Revista de intervención socioeducativa, 51, 27-44.

Black, P., \& Wiliam, D. (1998). Assessment and classroom learning. Assessment in Education: principles, policy \& practice, 5(1), 7-74.

Comisión Económica para América Latina y el Caribe (CEPAL). (2017). Derechos de las Personas Mayores: retos para la interdependencia y autonomía. Santiago: Naciones Unidas

Escuela de Psicología. (2017). Proyecto de Vinculación con el medio Universidad para Personas Mayores. Recuperado de http://www.ust.cl/vinculacion-conel-medio/sobre-vinculacion/proyecto-destacado/universidad-personasmayores/.

García Araneda, N. (2007). La educación con personas mayores en una sociedad que envejece. Horizontes Educacionales, 12 (2), 51-62.

García, A. J., \& Troyano Rodríguez, Y. (2010). Aprendizaje cooperativo en personas mayores universitarias. Estrategias de implementación en el Espacio Europeo de Educación Superior. Revista Interamericana de Educación de Adultos, 32(1). 6-21.

Granell, C. G., \& Salvador, C. C. (1994). De qué hablamos cuando hablamos de constructivismo. Cuadernos de pedagogía, 221, 8-10.

Monereo, C. (1999). Concepciones sobre el concepto de estrategias de aprendizaje y sobre su enseñanza. Madrid: Santillana.

Urzúa, A., \& Caqueo-Urízar, A. (2012). Calidad de vida: Una revisión teórica del concepto. Terapia psicológica, 30(1), 61-71.

Urzúa, A., Bravo, M., Ogalde, M., Vargas, C. (2011). Factores vinculados a la calidad de vida en la adultez mayor. Revista Médica de Chile, 139 (8), 1006-1014. 
Pensamiento y Acción Interdisciplinaria, Volumen 5, número 1, año 2019. ISSN 0719-8078. pp. 95-107

Dirección de correspondencia:

Sandra Sandoval. Psicóloga, UCN. Académica Universidad Santo Tomas

Contacto: ssandovalpasten@gmail.com. 